\title{
Modulação da cicatrização na cirurgia do glaucoma após aplicação de acetato de triancinolona subconjuntival isolado ou em associação à mitomicina C: estudo experimental
}

\section{Healing modulation in glaucoma surgery after application of subconjunctival triamcinolone acetate alone or combined with mitomycin C: an experimetal study}

Hayana Marques do Aragão Rangel ${ }^{1,2}$; Hévila Tamar Rolim²,3; Paula Vidigal ${ }^{4}$; Ivana Duval de Araújo ${ }^{5}$; Sebastião Cronemberger ${ }^{1}$

\author{
R E S U M O
}

\begin{abstract}
Objetivo: estudar a eficácia e segurança do uso de acetato de triancinolona subconjuntival isolado ou em associação à mitomicina C como modulador da cicatrização de trabeculectomias em coelhos. Métodos: trinta coelhos machos, albinos, raça Nova Zelândia foram submetidos à trabeculectomia bilateralmente. Os animais foram divididos em quatro grupos experimentais com 15 olhos por grupo: controle, mitomicina C, acetato de triancinolona e acetato de triancinolona + mitomicina C. Tonometria de aplanação e análise clínica da bolha através do Sistema de Graduação de Moorfields foram obtidas no pós-operatório. Para a avaliação da cicatrização, procedeu-se à análise quantitativa do infiltrado inflamatório (polimorfonucleares) através da coloração Hematoxilina \& Eosina e da proliferação vascular por imuno-histoquímica. Resultados: foi observada em todos os grupos diminuição significativa da pressão intraocular pós-operatória em relação à pré-operatória $(p<0,001)$. Contudo, não houve diferença entre os grupos $(p=0,186)$. 0 grupo acetato de triancinolona + mitomicina $C$ apresentou melhores índices na altura máxima da bolha e na vascularização da área central da bolha $(p=0,001)$; além disso, houve menor resposta inflamatória $(p=0,001)$ e menor proliferação vascular $(p=0,001)$ na fase intermediária do estudo em relação às monoterapias. Conclusão: a associação da mitomicina $C$ ao acetato de triancinolona resultou numa ação sinérgica entre esses agentes, com bolhas mais amplas e difusas e menor infiltrado inflamatório e menor proliferação vascular em estágio intermediário do acompanhamento neste modelo animal.
\end{abstract}

Descritores: Glaucoma. Cicatrização. Antimitóticos. Trabeculectomia. Procedimentos Cirúrgicos Oftalmológicos.

\section{INTRODUÇÃO}

( ) glaucoma é uma neuropatia óptica que causa cegueira irreversível, se não tratada adequadamente. É um problema de saúde pública, sendo a segunda maior causa de cegueira na população ${ }^{1}$. O objetivo de seu tratamento é a diminuição da pressão intraocular, através de medicações tópicas, procedimentos a laser e/ou cirurgias $^{2,3}$. Desde a primeira descrição, em 1968, a trabeculectomia tornou-se o procedimento padrão no tratamento cirúrgico do glaucoma. Tem como objetivo formar uma fistula que proporcione uma via alternativa para a drenagem do humor aquoso da câmara anterior para o espaço subconjuntival, a fim de reduzir a pressão intraocular $(\mathrm{PO})^{3,4}$.

Ao contrário de muitos tipos de cirurgia em que a cicatrização completa é o resultado desejável, a trabeculectomia visa a alcançar uma resposta parcial à cicatrização a fim de permitir a drenagem contínua do humor aquoso através da fistula4. A modulação da resposta à cicatrização na cirurgia do glaucoma é muitas vezes necessária, com o objetivo de alcançar a pressão adequada, maximizando o sucesso cirúrgico 5 . Há uma variedade de agentes disponíveis que podem modificar esta resposta.

\footnotetext{
1 - Universidade Federal de Minas Gerais, Departamento de Oftalmologia, Belo Horizonte, MG, Brasil. 2 - Santa Casa de Misericórdia de Belo Horizonte, Centro de Referência em Glaucoma e Catarata, Belo Horizonte, MG, Brasil. 3 - Universidade Federal de Rondônia, Departamento de Medicina, Porto Velho, RO, Brasil. 4 - Universidade Federal de Minas Gerais, Departamento de Patologia, Belo Horizonte, MG, Brasil. 5 - Universidade Federal de Minas Gerais, Departamento de Cirurgia, Belo Horizonte, MG, Brasil.
} 
Na prática oftalmológica, os antimetabólitos, 5-fluouracil (5-FU) e mitomicina C (MMC), revolucionaram a história da cirurgia do glaucoma, por isso são os fármacos mais utilizados na modulação da cicatrização ${ }^{4,6}$. Embora tenham impactado a taxa de sucesso cirúrgico na trabeculectomia, o mecanismo não específico desses agentes pode resultar em várias complicações oculares, como toxicidade corneal, bolhas avasculares, endoftalmite, hipotonia e, em alguns casos, são ineficazes ${ }^{7}$. Por conseguinte, ainda há a necessidade de estratégias alternativas para impedir a falência cirúrgica secundária ao excesso de cicatrização.

Agentes anti-inflamatórios já fazem parte da estratégia para modular a inflamação e a cicatrização de feridas no olho. Os hormônios esteroides podem ser vistos como protótipo 4 . Acetato de triancinolona (AT) é um corticoide em forma de suspensão injetável já utilizado para tratamento de doenças neovasculares, proliferativas e edematosas na oftalmologia. Seu potencial antiinflamatório é aproximadamente cinco vezes o do cortiso| $^{8,9}$. A ação anti-inflamatória e antifibrótica do AT é largamente mediada pela supressão da concentração de leucócitos e de sua funcionalidade, assim como pelos seus efeitos sobre a permeabilidade vascular. Isto leva a menor inflamação local, redução da liberação de fatores de crescimento e da produção de coágulo e fibrina. Como resultado, ocorre diminuição da atividade de fibroblastos e da resposta cicatricial 8,10

Apesar de já existirem alguns estudos avaliando o uso do AT na cirurgia do glaucoma, ainda há a necessidade de analisar se o AT pode ser utilizado em monoterapia ou apenas em associação à MMC. Por isso, nesta pesquisa, objetivou-se avaliar a eficácia e segurança do uso de AT isolado ou associado à MMC na trabeculectomia em coelhos.

\section{MÉTODOS}

\section{Modelo experimental}

Trinta coelhos machos, albinos, espécie Oryctolagus caniculus, raça Nova Zelândia, peso entre $2 \mathrm{~kg}$ e $3 \mathrm{~kg}$, foram incluídos no estudo. A Comissão de Ética em Animais da Universidade Federal de Minas Gerais aprovou esta pesquisa, e todos os animais foram tratados em concordância com os preceitos da Associação para Pesquisa em Visão e Oftalmologia (ARVO) - protocolo no CEUAV UFMG 149/2012.

Todos os animais foram submetidos à trabeculectomia em ambos os olhos, sob condições estéreis, pelo mesmo cirurgião, utilizando a mesma técnica. A anestesia geral foi feita com Cetamina $35 \mathrm{mg} / \mathrm{Kg}+$ Xilazina $5 \mathrm{mg} / \mathrm{Kg}+$ Acepromazina $0,75 \mathrm{mg} / \mathrm{Kg}$. Sutura corneana superior de tração foi feita com fio seda 6-0 para exposição do local a ser operado. Foi confeccionado retalho conjuntival base fórnice e retalho escleral retangular ( $3 \mathrm{~mm} \times 3 \mathrm{~mm})$ Em seguida, a esclerotomia e a iridectomia periférica foram realizadas. Procedeu-se ao fechamento escleral e conjuntival com duas suturas com fio mononylon 10-0. Ao final do procedimento, foi instilada uma gota de colírio de moxifloxacino 0,5\% e dexametasona 0,1\% associados.

Os animais foram distribuídos em quatro grupos experimentais de 15 olhos por grupo, já que cada animal foi operado bilateralmente e recebeu tratamentos diferentes em olhos direito e esquerdo. No grupo controle foi aplicada, através de esponja, $0,2 \mathrm{ml}$ de solução salina abaixo da cápsula de Tenon e sob retalho escleral por dois minutos. No grupo MMC, foi aplicada 0,4ml de mitomicina C a 0,03\% através de esponja, abaixo da cápsula de Tenon e sob retalho escleral por dois minutos. No grupo AT, foi administrado por via subconjuntival, $4 \mathrm{mg}$ 
de acetato de triancinolona superiormente à bolha ao final da cirurgia. E, no grupo AT+MMC, foram administrados 0,4ml de MMC a 0,03\% através de esponja, abaixo da cápsula de Tenon e sob retalho escleral por dois minutos e, por via subconjuntival, $4 \mathrm{mg}$ de acetato de triancinolona superiormente à bolha ao final da cirurgia.

\section{Análise clínica}

Os animais foram examinados no pré-operatório e nos dias um, três, sete, 14 e 30 após o procedimento cirúrgico. A tonometria de aplanação foi realizada com o Tonômetro de Perkins (Haag-Streit, Koniz, Switzerland), por meio de três medidas da Po, e a média foi analisada. O estudo da bolha fistulante foi baseado na avaliação fotográfica do local cirúrgico e na comparação com as fotos do sistema de classificação da bolha de Moorfields ${ }^{11}$. Esta análise fotográfica foi realizada por outro examinador que não conhecia os grupos.

\section{Análise imuno-histológica}

Cinco coelhos de cada grupo foram mortos e enucleados nos dias três, 14 e 30 para avaliação de marcadores de cicatrização, com pentobarbital intravenoso $(150 \mathrm{mg} / \mathrm{kg})$. O material foi corado com Hematoxilina \& Eosina (HE) para todos os animais e a região da conjuntiva operada foi avaliada para contagem de polimorfonucleares. Na análise imuno-histoquímica, foi utilizado o anticorpo VEGF (C-1): SC-7269 (Santa Cruz Biotechnology, Califórnia - USA) para investigar a expressão do VEGF A no endotélio vascular, avaliando a angiogênese. De cada lâmina foram retiradas três fotos em sequência da área relacionada, com aumento de 400 vezes, e, em seguida, foram avaliadas por patologista que também não conhecia os grupos.

\section{Análise estatística}

Foi feita utilizando o SPSS versão 19.0. Foram utilizados testes não paramétricos na análise clínica da bolha fistulante. A comparação entre grupos independentes foi realizada utilizando-se o teste de Kruskal-Wallis. E, na comparação entre as fases foi utilizado o teste de Friedman. Com o objetivo de avaliar a influência dos grupos e das fases do estudo na pressão intraocular foi utilizada a Análise de Variância baseada em um planejamento de Medidas Repetidas. A avaliação da influência do grupo e do tempo de avaliação até a morte dos animais (fase) nas variáveis imuno-histológicas, bem como da interação entre esses dois fatores (grupo e fase) foi realizada utilizando-se a técnica da Análise de Variância com dois fatores. Todos os resultados foram considerados significativos para uma probabilidade de significância inferior a 5\% $(p<0,05)$.

\section{RESULTADOS}

Não houve diferença significativa em relação ao peso inicial dos animais entre os grupos $(p=0,910)$. Em relação à pressão intraocular, em todos os grupos, os valores pré-operatórios foram maiores que os valores do pós-operatório final $(p=0,001)$. Contudo, houve diferença significativa entre os grupos apenas no 30 dia de pós-operatório (DPO): os grupos AT+MMC $(8,9 \pm 0,9 \mathrm{mmHg})$ e $M M C$ $(8,8 \pm 1,5 \mathrm{mmHg})$ apresentaram os menores índices pressóricos, seguidos pelo grupo AT $(9,9 \pm 1,1 \mathrm{mmHg})$ e depois pelo grupo controle $(11,4 \pm 1,5 \mathrm{mmHg})$ $(p=0,043)$ (Figura 1).

Após a avaliação da bolha pelo Sistema de Graduação da Bolha de Moorfields, nos critérios área central $(p=0,002)$ e máxima da bolha no 30 DPO 


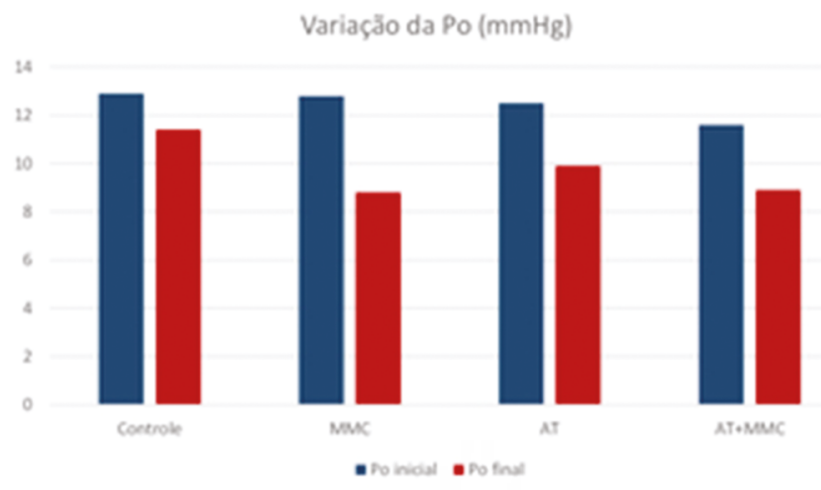

Figura 1. Comparação da pressão intraocular inicial e final.

houve melhores resultados nos grupos $A T+M M C$ $(2,8 \pm 0,4 ; 3,0 \pm 0,0)$ e $\operatorname{MMC}(2,8 \pm 0,4 ; 3,0 \pm 0,0)$. $\mathrm{Na}$ avaliação da altura máxima da bolha, o grupo AT+MMC $(1,8 \pm 0,4)$ apresentou melhores índices em relação aos outros grupos ( $P=0,001)$. Na análise da vascularização da área central, houve diferença significativa no $14^{\circ}$ DPO, com melhores resultados no grupo $A T+M M C(2,4 \pm 0,9) \quad(p=0,001)$. Já no critério vascularização da área máxima da bolha, no 14ํㅡㅁ DPO, os menores índices foram similares nos grupos AT+MMC $(2,4 \pm 0,5)$ e $\operatorname{MMC}(2,4 \pm 0,9)$ $(p=0,031)$ (Figura 2).

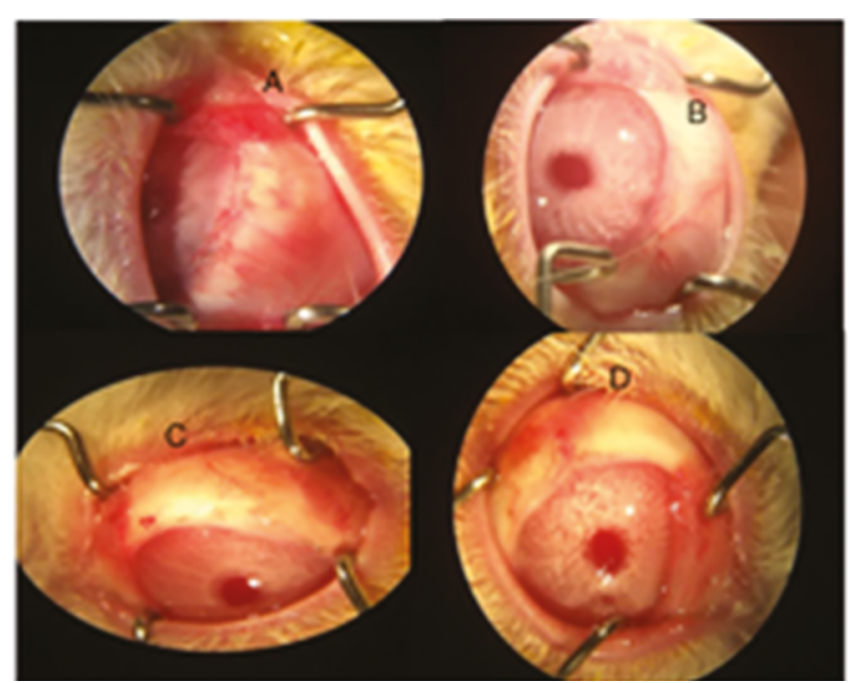

Figura 2. Fotografias do aspecto pós-operatório da trabeculectomia no sétimo DPO; Grupos: Controle (A), $M M C(B), A T(C)$ e $A T+M M C(D)$.
Houve poucas complicações durante o estudo, sem significância para os resultados. Dois casos de depósito esbranquiçado subconjuntival (um caso no grupo AT e outro no AT+MMC) que foram reabsorvidos ao final do acompanhamento.

$\mathrm{Na}$ análise quantitativa de polimorfonucleares ao longo do estudo, na fase inicial

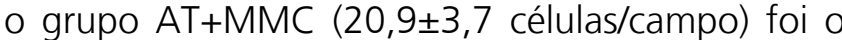
de menor proliferação celular, seguido pelo grupo AT $(22,1 \pm 2,3$ células/campo) $(p=0,001)$. Na fase intermediária, o grupo AT+MMC (18,0 1 1,9 células/ campo) permaneceu com melhores resultados, seguido pelo grupo MMC (19,3 $\pm 2,2$ células/campo) $(p=0,001)$. Já ao final do estudo, o grupo AT+MMC

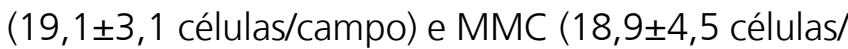
campo) apresentaram menor infiltrado inflamatório

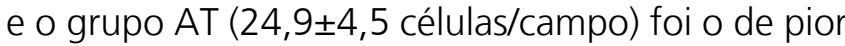
resultado ( $p=0,001)$ (Figuras 3 e 4$)$.

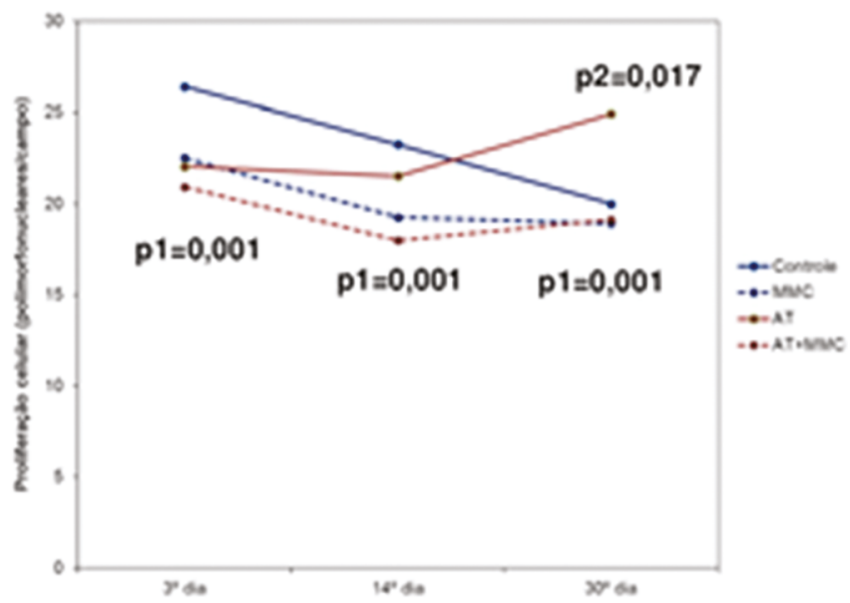

Figura 3. Contagem de polimorfonucleares ao longo do estudo; p1: comparação entre os grupos em cada fase; $p 2$ : comparação em cada fase por grupo.

A angiogênese, processo de crescimento de novos vasos sanguíneos, é um elemento chave da fase proliferativa da cicatrização. Sua avaliação mostrou, na fase inicial, melhores resultados no

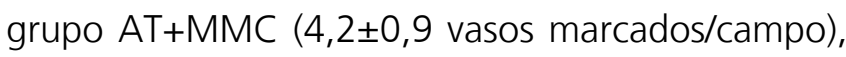




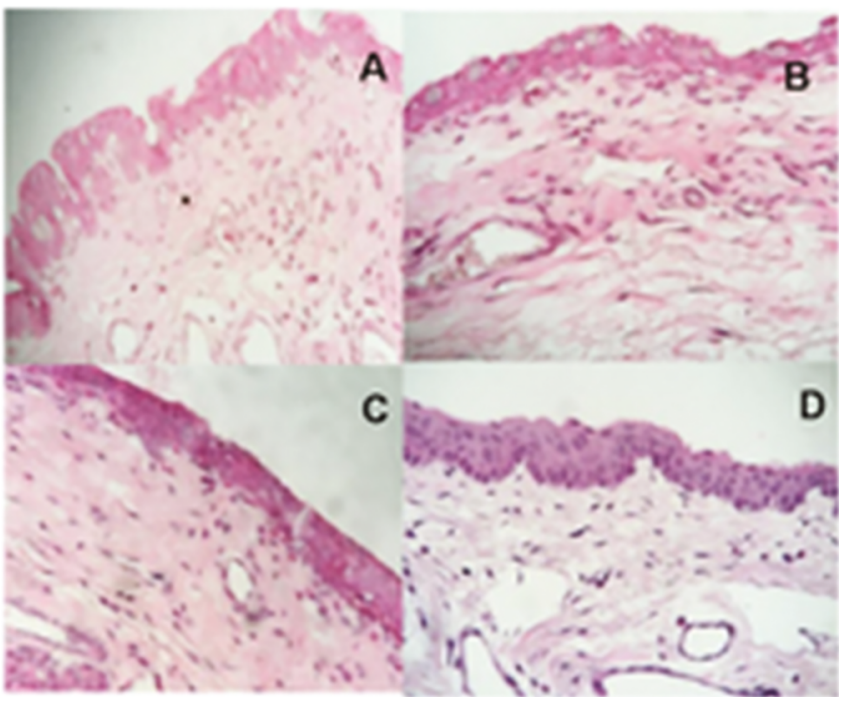

Figura 4. Cortes histológicos (HE) do globo ocular na região conjuntival de coelhos submetidos à cirurgia experimental analisando a contagem de polimorfonucleares no 14 DPO; Grupos: Controle (A); MMC (B); $A T(C) ; A T+M M C(D)$.

seguido pelo grupo MMC (5,4 $\pm 1,1$ vasos marcados/ campo) ( $p=0,001)$. No 14을 $=$ o grupo AT+MMC $(3,4 \pm 1,0$ vasos marcados/campo), permaneceu com menores índices de proliferação vascular $(P=0,001)$. Contudo, ao final da pesquisa o grupo com melhores resultados foi o grupo da $\mathrm{MMC}(4,1 \pm 1,2$ vasos marcados/campo) ( $p=0,001)$ (Figuras 5 e 6).

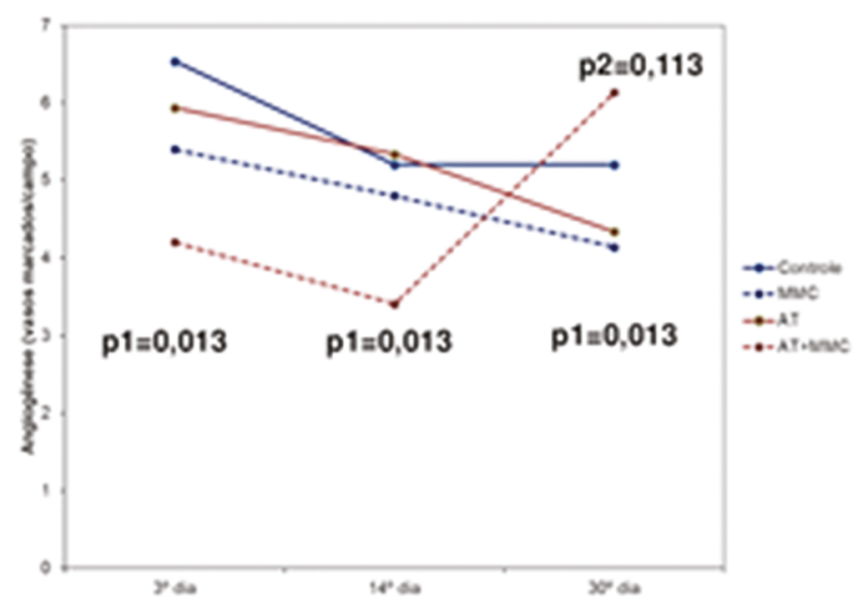

Figura 5. Resultados da proliferação vascular ao longo do estudo; p1: comparação entre os grupos em cada fase; p2: comparação em cada fase por grupo.

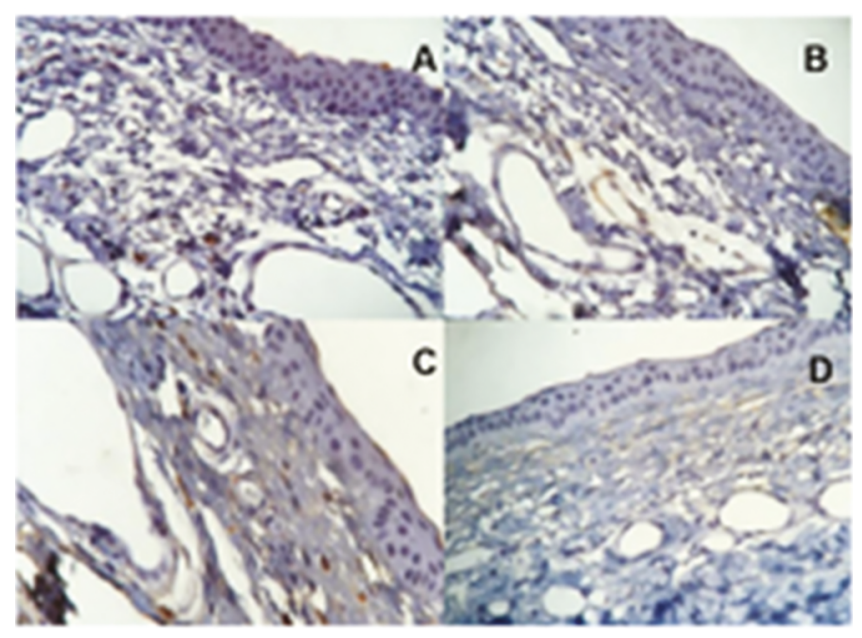

Figura 6. Cortes histológicos (avaliação imuno-histoquímica) da conjuntiva de coelhos submetidos à cirurgia experimental, analisando a deposição do cromógeno marrom no endotélio

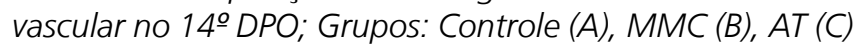
e $A T+M M C(D)$.

\section{DISCUSSÃO}

A cascata de cicatrização é um evento fisiológico, complexo e dinâmico que compreende uma cascata de diferentes processos intimamente ligados e ainda não totalmente compreendidos ${ }^{5}$. Para que haja sucesso em longo prazo na cirurgia fistulante de glaucoma, a cicatrização completa não pode ocorrer. Por isso, a maior limitação da trabeculectomia, é o desenvolvimento de fibrose na conjuntiva e episclera secundário à proliferação de fibroblastos e depósito de colágeno no local da fístula ${ }^{12}$. Foram avaliados neste estudo o modulador padrão, a mitomicina $C$, e um modulador alternativo, o acetato de triancinolona, isolados ou em associação em modelo experimental de coelhos.

Houve menores índices pressóricos ao final do estudo nos grupos AT+MMC e MMC, mas sem diferença entre eles. Contudo não ocorreu aumento pressórico nos grupos que utilizaram o acetato de triancinolona. A utilização deste anti-inflamatório 
na oftalmologia é associada à elevação da pressão intraocular induzida pelo uso de corticoide e sua injeção subconjuntival pode causar este aumento por vários meses. Acredita-se que isto ocorra secundariamente à redução na facilidade de escoamento do humor aquoso pela malha trabecular pelo depósito das partículas do fármaco no trabeculado9,13. Os resultados do presente estudo estão em concordância com Hogewind et al. ${ }^{8}$ que utilizaram 20mg de AT em seu estudo, também sem aumento pressórico, sugerindo que o efeito no aumento da pressão induzido pelo corticoide na malha trabecular é compensado pela diminuição pressórica secundária à trabeculectomia.

O primeiro relato da utilização do AT como adjuvante na cirurgia do glaucoma foi feito por Giangiacomo et al. ${ }^{14}$, em 1986, que relataram $87,5 \%$ de sucesso cirúrgico e bolhas difusas ao final do acompanhamento, sugerindo efeito positivo do AT na modulação da cicatrização. No presente estudo, quando o AT foi utilizado em monoterapia não demostrou bons resultados em relação à associação e à MMC isolada, em discordância com Hogewind, que apresentou taxa de sucesso cirúrgico similar entre os grupos no acompanhamento de cinco anos, sugerindo que AT isolado poderia ser usado com adjuvante na cirurgia fistulante ${ }^{8}$. Esta diferença de resultados é justificada pela maior dosagem utilizada no estudo.

A maioria das publicações avaliou o uso da associação AT e MMC. Tham et al. ${ }^{15}$, em série de casos, injetaram AT (1,2mg) diretamente na ampola filtrante de três olhos submetidos à facotrabeculectomia, de três à trabeculectomia com $M M C$, e de cinco à revisão de trabeculectomia com 5-FU. Relataram diminuição da Po sem necessidade de medicação até três meses e não observaram sinais de perda endotelial ou progressão da catarata. Em 2009, outra série de casos avaliou o uso de AT retrobulbar (20mg) nas trabeculectomias com MMC. Apresentaram diminuição significativa da Po em seis meses $(P<0,001)$ sem necessidade de medicação hipotensora e não houve complicações (infecção, hipotonia, ulceração da conjuntiva) ${ }^{16}$. Apesar de serem apenas relatos de casos, estes resultados corroboram para o efeito positivo da associação desses moduladores na trabeculectomia.

Já em estudo, prospectivo, randomizado e controlado, avaliou-se a eficácia da injeção de AT subtenoniana (20mg) no sucesso das trabeculectomias (com MMC) em glaucomas secundários. Sucesso completo foi de $65,4 \%$ no grupo do AT e $63 \%$ no grupo controle $(p=0,77)$. As características da ampola fistulante foram similares em ambos os grupos $(p>0,40)$ e não houve diferenças significativas de Po no seguimento $(p>0,05)^{17}$. Os achados deste estudo foram similares, quando AT foi associado à MMC nas trabeculectomias, à recente série retrospectiva de casos que analisou a administração de AT intracameral $(0,1-0,3 \mathrm{ml}$, $4 \mathrm{mg} / \mathrm{ml}$ ). O sucesso completo foi de $68,4 \%$ no grupo estudado e $52,4 \%$ no grupo controle, já o sucesso parcial foi de $31,6 \%$ e $47,6 \%$, respectivamente. Também não houve aumento das complicações no grupo da AT $(p>0.05)^{18}$. Esses resultados estão em concordância com os encontrados no vigente estudo que mostraram diminuição pressórica nos pós-operatório e bom aspecto clínico da trabeculectomia no grupo AT+MMC, mas com resultados similares ao grupo que utilizou $M M C$ isolado.

Não identificamos estudos prévios relatando o efeito na cascata de cicatrização do AT e AT+MMC na trabeculectomia em coelhos. Em 
estudo experimental que utilizou outro corticoide, a dexametasona em forma de implante intravitreo, como adjuvante à trabeculectomia, houve menor depósito de colágeno e maior sobrevida da cirurgia em relação ao grupo controle, contudo o grupo da MMC apresentou melhores resultados ${ }^{19}$. Esses resultados em associação aos presentes achados reforçam a ação positiva dos corticoides na modulação da cicatrização na cirurgia do glaucoma, contudo, tanto a dexametasona quanto o acetato de triancinolona não foram superiores ao antifibrótico padrão, a MMC. Entretanto, não houve avaliação da associação dos adjuvantes, como no vigente estudo, que analisou a associação AT+MMC, mostrando maior modulação da cicatrização do que estes agentes isolados nas fases inicial e intermediária, tanto em relação ao infiltrado inflamatório quanto à resposta vascular. Isto é corroborado por relatos de que o uso tópico de corticoide no pósoperatório aumenta significativamente o sucesso cirúrgico por inibição da cicatrização de feridas como resultado da supressão da inflamação e proliferação de fibroblastos ${ }^{20}$, assim como, também por pesquisa em seres humanos, através de cultura de fibroblasto da cápsula de Tenon, que mostraram que os corticoides inibem a ligação e a proliferação celular $^{21}$. Contudo, o resultado não promissor do AT isolado e o bom resultado do AT+MMC apenas até a fase intermediária neste modelo animal pode ser justificado pela baixa dosagem de $4 \mathrm{mg}$ deste corticoide.
Esta pesquisa não avaliou diretamente o tempo de sobrevida da bolha filtrante, critério utilizado em alguns estudos experimentais, definido por uma aparência cicatrizada, plana e vascularizada da bolha. Contudo, a avaliação clínica utilizando o Sistema de Graduação da Bolha de Moorfields analisou a extensão, altura e grau de vascularização do local da cirurgia, e a má pontuação desses parâmetros, indiretamente, apontaria para o critério de sobrevida. Outra limitação foi o número pequeno de olhos na fase final do estudo, mas este número é semelhante ao de publicações prévias ${ }^{12,22}$.

Devido à natureza multifatorial da cicatrização de feridas, é provável que múltiplos agentes, usados simultaneamente ou sequencialmente, possam ser necessários para a modulação ideal. A associação da MMC ao acetato de triancinolona, nos resultados vigentes, aponta para ação sinérgica entre esses agentes, com bolhas mais amplas e difusas, menor infiltrado inflamatório e menor proliferação vascular em estágio intermediário do acompanhamento neste modelo animal.

Os resultados satisfatórios do presente estudo como também os já publicados em relação ao uso de AT como adjuvante às trabeculectomias devem, no entanto, ser considerados com cautela. Ainda são necessários ensaios clínicos prospectivos multicêntricos de longo acompanhamento com objetivo de avaliar, dose e via de administração ideais que produzam o melhor resultado cirúrgico com mínimos efeitos colaterais. 


\title{
A B S T R A C T
}

\begin{abstract}
Objective: to study the efficacy and safety of the use of subconjunctival triamcinolone acetate alone or in combination with mitomycin C as a modulator of trabeculectomy healing in rabbits. Methods: we submitted thirty male, albino, New Zealand rabbits to bilateral trabeculectomy. We divided the animals into four experimental groups with 15 eyes per group: control, mitomycin C, triamcinolone acetate and triamcinolone acetate + mitomycin C. We performed aplanation tonometry and clinical analysis of the bleb through the Moorfields Graduation System in the postoperative period. For the evaluation of healing, we carried out the quantitative analysis of the inflammatory infiltrate (polymorphonuclear) through Hematoxylin \& Eosin staining, and vascular proliferation, through immunohistochemistry. Results: we observed a significant decrease in postoperative intraocular pressure in all groups compared with the preoperative pressure $(p<0.001)$. However, there was no difference between groups $(p=0.186)$. The triamcinolone + mitomycin $C$ acetate group presented better indices as for the maximum bleb height and vascularization of the bleb central area ( $p=0.001)$; in addition, there was a lower inflammatory response $(p=0.001)$ and lower vascular proliferation $(p=0.001)$ in the intermediate phase of the study compared with the monotherapies. Conclusion: the combination of mitomycin $C$ and triamcinolone acetate resulted in a synergistic action between these agents, with broader and more diffuse blebs, less inflammatory infiltrate and less vascular proliferation in the intermediate stages of follow-up in this animal model.
\end{abstract}

Keywords: Glaucoma. Wound Healing. Antimitotic Agents. Trabeculectomy. Ophthalmologic Surgical Procedures.

\section{REFERÊNCIAS}

1. Hennis A, Wu SY, Nemesure B, Honkanen R, Leske MC; Barbados Eyes Studies Group. Awareness of incident open-angle glaucoma in a population study: the Barbados Eye Studies. Ophthalmology. 2007;114(10):1816-21.

2. Harasymowycz P, Birt C, Gooi P, Heckler L, Hutnik C, Jinapriya $D$, et al. Medical management of glaucoma in the 21st century from a Canadian perspective. J Ophthalmol 2016;2016:6509809.

3. Razeghinejad MR, Fundemberg SJ, Spaeth GL. The changing conceptual basis of trabeculectomy: a review of past and current surgical techniques. Surv Ophthalmol. 2012;57(1):1-25.

4. Lama PJ, Fechtner RD. Antifibrotics and wound healing in glaucoma surgery. Surv Ophthalmol. 2003;48(3):314-46.

5. Cordeiro MF, Chang L, Lim KS, Daniels JT, Pleass $R D$, Siriwardena $D$, et al. Modulating conjuctival wound healing. Eye (Lond). 2000;14(Pt 3B):536-47.

6. Lockwood A, Brocchini S, Khaw PT. New developments in the pharmacological modulation of wound healing after glaucoma filtration surgery. Curr Opin Pharmacol. 2012;13(1):65-71.

7. Van Bergen T, Van de Velde S, Vandewalle E, Moons L, Stalmans I. Improving patient outcomes following glaucoma surgery: state of the art and future perspectives. Clin Ophthalmol. 2014;8:85767.
8. Hogewind BF, Pijl B, Hoyng CB, Theelen T. Purified triamcinolone acetonide as antifibrotic adjunct in glaucoma filtering surgery. Graefes Arch Clin Exp Ophthalmol. 2013;251(4):1213-8.

9. Kalina PH, Erie JC, Rosenbaum L. Biochemical quantification of triamcinolone in subconjunctival depots. Arch Ophthalmol. 1995;113(7):867-9.

10. Hosseini $H$, Mehryar $M$, Farvardin $M$. Focus on triamcinolone acetonide as an adjunct to glaucoma filtration surgery. Med Hypotheses. 2007;68(2):4013.

11. Wells AP, Crowston JG, Marks J, Kirwan JF, Smith $G$, Clarke JC, et al. A pilot study of a system for grading of drainage blebs after glaucoma surgery. J Glaucoma. 2004;13(6):454-60.

12. Memarzadeh F, Varma R, Lin LT, Parikh JG, Dustin L, Alcaraz A, et al. Postoperative use of bevacizumab as an antifibrotic agent in glaucoma filtration surgery in the rabbit. Invest Ophthalmol Vis Sci. 2009;50(7):3233-7.

13. Zaka-ur-Rab S, Mahmood S, Shukla M, Zakir SM, Khan BA, Owais M. Systemic absorption of triamcinolone acetonide after posterior sub-Tenon injection. Am J Ophthalmol. 2009;148(3):414-9.

14. Giangiacomo J, Dueker DK, Adelstein E. The effect of preoperative subconjunctival triamcinolone administration on glaucoma filtration. I. Trabeculectomy following subconjunctival triamcinolone. Arch Ophthalmol. 1986;104(6):83841. 
15. Tham CC, Li FC, Leung DY, Kwong YY, Yick DW, Chi CC, et al. Intrableb triamcinolone acetonide injection after bleb-forming filtration surgery (trabeculectomy, phacotrabeculectomy, and trabeculectomy revision by needling): a pilot study. Eye (Lond). 2006;20(12):1484-6.

16. Kahook MY, Camejo L, Noecker RJ. Trabeculectomy with intraoperative retrobulbar triamcinolone acetonide. Clin Ophthalmol. 2009;3:29-31.

17. Yuki K, Shiba D, Kimura I, Ohtake Y, Tsubota K. Trabeculectomy with or without intraoperative sub-tenon injection of triamcinolone acetonide in treating secondary glaucoma. Am J Ophthalmol. 2009;147(6):1055-60.

18. Alagöz N, Alagöz C, Yildirim Y, Yesilkaya C, Altan Ç, Bozkurt $E$, et al. The effect of adjuvant intracameral triamcinolone acetonide on the surgical results of trabeculectomy with mitomycin C. Turk J Ophthalmol. 2016;46(4):169-74.

19. Kiddee W, Orapiriyakul L, Kittigoonpaisan K,Tantisarasart T, Wangsupadilok B. Efficacy of adjunctive subconjunctival bevacizumab on the outcomes of primary trabeculectomy with mitomycin C: a prospective randomized placebocontrolled trial. J Glaucoma. 2015;24(8):600-6.
20. Araujo SV, Spaeth GL, Roth SM, Starita RJ. A tenyear follow-up on a prospective, randomized trial of postoperative corticosteroids after trabeculectomy. Ophthalmology. 1995;102(12):1753-9.

21. Nguyen KD, Lee DA. Effect of steroids and nonsteroidal anti-inflammatory agents on human ocular fibroblast. Invest Ophthalmol Vis Sci 1992;33(9):2693-701.

22. Cheng G, Xiang $H$, Yang G, Ma J, Zhao J. Bevacizumab inhibits angiogenesis and inflammation in rat filtration surgery model. Cell Biochem Biophys 2015;73(1):71-7.

Recebido em: 10/03/2018

Aceito para publicação em: 10/05/2018

Conflito de interesse: nenhum.

Fonte de financiamento: nenhuma.

\section{Endereço para correspondência:}

Hayana Marques do Aragão Rangel

E-mail: hayanarangel@yahoo.com.br hevilarolim@unir.br 